\title{
Attitudes and Response to a Smartphone-Based Digital Pill Intervention to Enhance PrEP Adherence Among Men Who Have Sex With Men With Stimulant Use
}

Georgia Goodman ${ }^{1,2}$, BS; Conall O'Cleirigh ${ }^{1,2}$, PhD; Kenneth H Mayer ${ }^{2,3}$; Edward W Boyer ${ }^{2,4}$; Rochelle K Rosen ${ }^{5,6}$; Peter R Chai ${ }^{2,4}$

\footnotetext{
${ }^{1}$ Department of Psychiatry, Massachusetts General Hospital / Harvard Medical School, Boston, MA, United States

${ }^{2}$ The Fenway Institute, Fenway Health, Boston, MA, United States

${ }^{3}$ Infectious Diseases, Beth Israel Deaconess Medical Center / Harvard Medical School, Boston, MA, United States

${ }^{4}$ Department of Emergency Medicine, Brigham and Women's Hospital, Boston, MA, United States

${ }^{5}$ Centers for Behavioral and Preventive Medicine, The Miriam Hospital, Providence, RI, United States

${ }^{6}$ Department of Behavioral and Social Sciences, School of Public Health, Brown University, Providence, RI, United States
}

\section{Corresponding Author:}

Georgia Goodman, BS

Massachusetts General Hospital / Harvard Medical School

Department of Psychiatry

1 Bowdoin Square

Boston, MA

United States

Phone: 8573136722

Email: ggoodman@mgh.harvard.edu

\section{Abstract}

Background: Digital pills contain a radiofrequency emitter and gelatin capsule that over-encapsulate a study medication. The radiofrequency emitter, activated by the chloride ion gradient in the stomach, transmits a signal to a wearable Reader device following ingestion. The Reader relays ingestion data to a smartphone app and cloud-based server, allowing for real-time verification of ingestion events and adherence measurement. Digitized pre-exposure prophylaxis (PrEP) may improve outcomes in populations with suboptimal adherence related to substance use. Stimulant use is particularly prevalent among men who have sex with men (MSM) and is associated with increased HIV risk behavior and transmission. We conducted seven focus groups with MSM who use stimulants $(\mathrm{N}=16)$ to inform the development of PrEPsteps, a novel smartphone-based adherence intervention respondent to data from digitized PrEP and designed to augment adherence.

Objective: To inform the specification of the design, content, and delivery of the smartphone-based PrEPsteps adherence system via focus groups with HIV-negative MSM who use stimulants.

Methods: Seven focus groups were conducted with HIV-negative MSM reporting stimulant use (eg, cocaine, methamphetamine) in the past six months. Participants self-reported medical history, substance use and sexual activity. Focus groups explored responses to digital pill technology, willingness and barriers to use, content and timing of adherence messaging, and three intervention components: (1) abbreviated cognitive behavioral therapy (CBT) adherence counseling (LifeSteps); (2) contingent reinforcement/corrective feedback; and (3) substance use-related Screening, Brief Intervention and Referral to Treatment (SBIRT). Focus groups were transcribed and analyzed using applied thematic analysis.

Results: Sixteen individuals participated in focus groups. All were male; age ranged from 24 to 63 (mean 39.9, SD 14.1) and most self-identified as gay $(\mathrm{N}=13)$. Participants were primarily non-Hispanic or Latino $(\mathrm{N}=12)$; 10 identified as white, two as black, and four as multiracial. Most had at least a college degree $(\mathrm{N}=14)$. Twelve participants were taking PrEP, 5 of whom

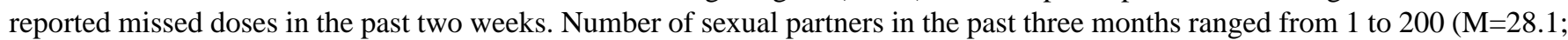
$\mathrm{SD}=50.9$ ). Fifteen participants reported using stimulants during the last 30 days. Participants viewed digital pills as a tool to enhance PrEP adherence and accountability. They expressed a willingness to use the digital pill and identified physicians, family members, and partners as people with whom they would share adherence data. The Reader was viewed as the most difficult technological component to use, although participants also described the device as itself an adherence reminder. Participants identified customizability as a valuable aspect of the technology; message and reminder content, structure, and scheduling were 
all considered customizable. With regard to the intervention, participants were accepting of and willing to interact with corrective feedback messages linked to a brief CBT LifeSteps booster session.

Conclusions: PrEPsteps, a smartphone-based adherence intervention, was viewed as acceptable by HIV-negative MSM who use stimulants. Individuals perceived corrective feedback notifications to be the most helpful component of the system, and expressed a strong preference for customizability across the intervention.

(iproc 2019;5(1):e15273) doi: $10.2196 / 15273$

\section{KEYWORDS}

focus groups; HIV; medication adherence; prep; MSM

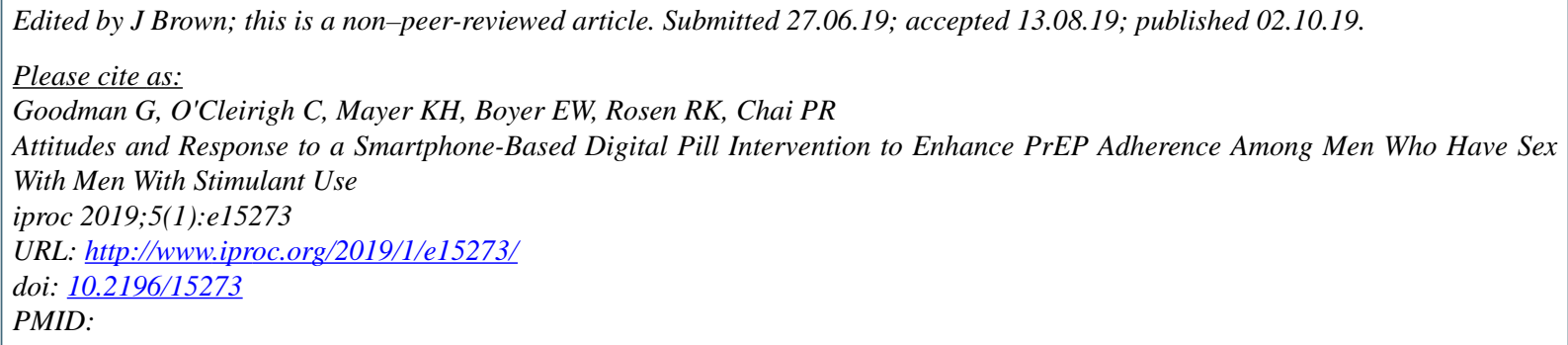

CGeorgia Goodman, Conall O'Cleirigh, Kenneth H. Mayer, Edward W. Boyer, Rochelle K. Rosen, Peter R. Chai. Originally published in Iproceedings (http://www.iproc.org), 02.10.2019 This is an open-access article distributed under the terms of the Creative Commons Attribution License (https://creativecommons.org/licenses/by/4.0/), which permits unrestricted use, distribution, and reproduction in any medium, provided the original work, first published in Iproceedings, is properly cited. The complete bibliographic information, a link to the original publication on http://www.iproc.org/, as well as this copyright and license information must be included. 